\title{
Uma Ontologia para Apoio à Recomendação Automática e Personalizada de Conteúdo Considerando Estilos de Aprendizagem de Estudantes em Sistemas Adaptativos para Educação
}

\author{
Vitor C. de Carvalho ${ }^{1}$, Miller M. Mendes ${ }^{1}$, Hiran N. M. Ferreira ${ }^{2}$ \\ Fabiano A. Dorça ${ }^{1}$, Renan G. Cattelan ${ }^{1}$ \\ ${ }^{1}$ Faculdade de Computação - Universidade Federal de Uberlândia (UFU) \\ Uberlândia - MG - Brasil. \\ ${ }^{2}$ Instituto Federal de Educação, Ciência e Tecnologia do Sul de Minas Gerais \\ (IFSULDEMINAS) \\ Passos - MG - Brasil \\ millerdcomp.ufu.br, vitoramestrado.ufu.br \\ hiran.ferreira@ifsuldeminas.edu.br, \{renan, fabianodor\}@ufu.br
}

\begin{abstract}
Several approaches to personalized recommendation of content in adaptive systems for education have emerged. Many of them have considered the importance of taking into account students' learning styles in order to achieve better results in the learning process. Recent studies confirm this trend. Thus, this work aims to present an ontology to support these approaches, using as a basis the IEEE LOM and the model of Felder-Silverman for learning styles. As a result, the proposed ontology generates a vectorial representation of learning objects considering its teaching styles. The ontology has been validated through experiments, and promising results were obtained, demonstrating the potencial contribution of the proposal to adaptive systems for education.
\end{abstract}

Resumo: Diversas abordagens para recomendação personalizada de conteúdo em sistemas adaptativos para educação têm surgido. Muitas têm congitado a importância de levar em consideração os estilos de aprendizagem do estudante para que melhores resultados possam ser alcançados no processo de aprendizagem. Estudos recentes comprovam essa tendência. Desta forma, este trabalho tem como objetivo apresentar uma ontologia para apoio à essas abordagens, usando como base o padrão IEEE LOM e o modelo de Felder-Silverman. Como resultado, a ontologia proposta gera uma representação vetorial de objetos de aprendizagem considerando os estilos de aprendizagem satisfeitos por eles. A ontologia foi validada através de experimentos, e resultados promissores foram obtidos, demonstrando o potencial de contribuição desta proposta a sistemas adaptativos para educação.

\section{Introdução}

A web pode ser vista como uma grande biblioteca virtual, onde com pouco tempo de busca, é possível se encontrar todo tipo de conteúdo. Porém, a forma como ela foi construída inicialmente dava possibilidade para que apenas os seres humanos pudessem entender o conteúdo apresentado. Para que sistemas computacionais processassem os dados 
V Congresso Brasileiro de Informática na Educação (CBIE 2016)

Anais do XXVII Simpósio Brasileiro de Informática na Educação (SBIE 2016)

da web, criou-se a Web Semântica. O seu surgimento trouxe tecnologias e conceitos que podem ser aproveitados em diversas áreas de pesquisa.

Entre as tecnologias destacadas temos as ontologias, que por definição, são formas que especificam e descrevem conceitos, bem como as relações que existem ente eles [Gruber 1993]. Em trabalhos voltados a educação, ontologias podem ser utilizadas para representar modelos de estudantes ou, ainda, de Objetos de Aprendizagem(OAs) [Wiley 2002]. OAs são recursos virtuais de aprendizagem, como vídeos, imagens e textos que tem por objetivo trazer informação e conteúdo a ser assimilado por um aprendiz. Porém, para que seja possível se utilizar um OA, é necessário que este possua um arquivo de descrições de seus dados, os chamados metadados.

Existem diversos padrões de metadados, como o IEEE LOM [IEEE 2002], utilizado neste trabalho, que garantem que os OAs possuem algumas características importantes, como a Interoperabilidade, a Acessibilidade e a Durabilidade. Em geral, estes metadados são construídos em arquivos XML, porém, é possível modelar o padrão IEEE LOM em uma ontologia. Ao modelar os metadados em uma ontologia, temos a posse de uma rede semântica rica em informações que nos oferece suporte a geração de regras de inferência poderosas em linguagens como Semantic Web Rule Language (SWRL) e Semantic Query-Enhanced Web Rule Language (SQWRL).

Neste trabalho, denominamos como vetorização de um OA, a sua representação através de um vetor que mostra explicitamente sua relação com os Estilos de Aprendizagem (EAs) que ele atende. Para isto, utilizamos o modelo proposto por [Felder and Silverman 1988] e construímos uma ontologia que recebe os metadados de um OA no padrão IEEE LOM e automaticamente gera sua representação vetorial em relação aos EAs que atende. Isso foi feito através de regras de inferência geradas a partir do mapeamento entre o IEEE LOM e o modelo Felder de EAs, possibilitando, então, sua recomendação a estudantes, considerando seus EAs específicos.

O restante deste artigo está organizado da seguinte maneira: na Seção 2, são discutidos trabalhos relacionados; na Seção 3, são apresentados os principais conceitos utilizados na proposta; na Seção 4, é descrita a abordagem proposta e alguns exemplos de sua utilização e resultados; a Seção 5 apresenta um experimento e, por fim, na Seção 6, são feitas as considerações finais.

\section{Trabalhos Relacionados}

O número de pesquisas que aproveitam dos recursos da Web Semântica para educação vem crescendo a cada dia. O trabalho de Rezende et al. 2015, por exemplo, propõe uma Ontologia para o perfil do estudante com objetivo de facilitar a recomendação de conteúdo em sistemas de ensino.

Em seu sistema, Rezende et al. 2015 utilizaram da modelagem dos estudantes por informações destacadas como relevantes em outros trabalhos e modelam também o contexto em que o aluno se encontrava. Para a ontologia que implementa este modelo, foi dada o nome de PERSONNA e esta foi integrada à arquitetura que denominaram BROAD-RS.

Dorça and Resende 2015 listaram em seu trabalho 16 combinações de EAs, apresentando como separar os estudantes em grupos, levando em consideração seus EAs. 
V Congresso Brasileiro de Informática na Educação (CBIE 2016)

Anais do XXVII Simpósio Brasileiro de Informática na Educação (SBIE 2016)

Alternativamente, temos o trabalho de Ferreira et al. 2015 que divide os estudantes em grupos baseados em seu contexto, através de dados obtidos por tecnologia de Computação Ubíqua e, assim, recomendam seus objetos de aprendizagem.

Gluz and Vicari 2011 propõem uma Ontologia para representação de Metadados nos padrões IEEE LOM, DublinCore [Weibel et al. 1998] e o OBAA [Vicari et al. 2010]. Porém, este estudo não oferece recursos além da representação destes metadados, deixando em aberto as possíveis funcionalidades existentes.

Este trabalho se destaca devido a ontologia criada e as ferramentas disponibilizadas em tecnologias de inferência, podendo agregar eficiência naqueles que necessitam representar seus OAs de uma forma mais próxima ao modelo do estudante. O que traz a possibilidade de realizar uma recomendação automática e eficiente dos OAs de acordo com os EAs dos estudantes.

A abordagem apresentada traz uma tentativa dessa aproximação, utilizando dos Estilos de Aprendizagem que devem estar presentes ao modelo do estudante. Mostra, assim, como utilizar dos OAs em uma representação vetorial baseada nos EAs, automatizando de forma eficiente os processos de recomendação, considerando os EAs de cada estudante, os metadados e OAs disponíveis.

\section{Fundamentação Teórica}

Serão apresentados nesta seção, conceitos necessários à compreensão deste trabalho.

\subsection{Modelo de Felder-Silverman}

O trabalho realizado por Felder and Silverman 1988 identificou que cada estudante tem sua forma de receber e processar informações com eficiência. A essas características foram dadas o nome de Estilos de Aprendizagem.

O modelo original é composto por quatro dimensões, cada uma possuindo dois EAs, conforme apresentado na Tabela 1. EAs não são mutuamente exclusivos, sendo que indivíduo pode ser representado pela combinação de um estilo para cada uma das quatro dimensões. Este modelo é muito usado por dar a base para representar o perfil dos estudantes em Sistemas Adaptativos e Inteligentes para Educação (SAIE) [Graf et al. 2007].

Dentre os motivos para o uso frequente deste modelo de EAs, pode-se destacar o fato de ser baseado em extensa experimentação [Dorça et al. 2013]. Graf et al. 2009 relata também que os EAs podem ser usados para melhorar o modelo de aluno em sistemas educacionais para fornecer adaptabilidade mais precisas e holísticas.

\subsection{Padrão IEEE Learning Object Metadata}

Todo OA deve possuir importantes atributos, são eles: Interoperabilidade, Acessibilidade e Durabilidade. Interoperabilidade é a capacidade de se usar um OA feito em sistemas diferentes daqueles para qual foram criados. Acessibilidade da a capacidade dos OAs de serem utilizados remotamente e, Durabilidade, que é a possibilidade de utilizar um OA, sem reprojeto ou recriação, mesmo quando a base tecnológica muda.

Para que essas características se mantenham, é necessário que haja um padrão, como o LOM [IEEE 2002]. O LOM é composto por 68 elementos hierarquizados, divididos em 9 categorias, que descrevem a semântica dos metadados de um OA. Neste 
V Congresso Brasileiro de Informática na Educação (CBIE 2016)

Anais do XXVII Simpósio Brasileiro de Informática na Educação (SBIE 2016)

Tabela 1. Tabela de estilos do Modelo Felder-Silverman (adaptada de Franzoni et al. 2008).

\begin{tabular}{|c|c|c|}
\hline $\begin{array}{c}\text { Dimensao do Estilo de } \\
\text { Apredizagem }\end{array}$ & Tipo & Descrição \\
\hline \multirow{2}{*}{ Percepção } & Sensitivo (S) & $\begin{array}{l}\text { Sabe lidar com fatos, dados puros e experimentos, é paciente com } \\
\text { os detalhes, mas não gosta de complicaçốes. }\end{array}$ \\
\hline & Intuitivo (I) & $\begin{array}{l}\text { Sabe lidar com principios e teorias, fica facilmente entediado } \\
\text { quando são apresentados muitos detalhes e tende a aceitar } \\
\text { complicações. }\end{array}$ \\
\hline \multirow{2}{*}{ Canal de Entrada } & Visual (Vi) & $\begin{array}{l}\text { Se lembra bem do que viu: imagens, diagramas, tabelas temporais, } \\
\text { filmes, etc. }\end{array}$ \\
\hline & Verbal (Ve) & Se lembra do que escutou, leu ou disse. \\
\hline \multirow{2}{*}{ Processamento } & Ativo (A) & Aprende bem em grupo e com o material que tem em mãos. \\
\hline & Reflexivo (Re) & $\begin{array}{l}\text { Aprende melhor quando pensa e reflete sobre a informação dada a } \\
\text { ele. Trabalha melhor sozinho ou com no máximo mais uma pessoa. }\end{array}$ \\
\hline \multirow{2}{*}{ Entendimento } & Sequencial (Seq) & $\begin{array}{c}\text { Segue um raciocínio linear quando está resolvendo problemas e } \\
\text { pode trabalhar com material especifico uma vez que compreende } \\
\text { ele parcialmente ou superficialmente. }\end{array}$ \\
\hline & Global (G) & $\begin{array}{l}\text { Tem grandes intuições sobre a informação, pode ter dificuldade de } \\
\text { expor como chegou a certo resultado, precisa de uma visão geral. }\end{array}$ \\
\hline
\end{tabular}

trabalho, utilizou-se 5 desses elementos, são eles: Structure, Format, Interactivity Type, Leaning Resource Type e Interactivity Level.

Structure é o campo que oferece uma descrição da estrutura organizacional do OA. Format nos mostra o tipo de dado trabalhado pelo OA para facilitar a identificação de quais softwares serão necessários no seu acesso. Os tipos permitidos pelo campo Format são definidos pelo registro IANA de tipos MIME(ver RFC2048:1996 [Borenstein and Freed 1996]).

Interactivity Type fornece informação do tipo predominante de aprendizado no OA (Ativo ou Expositivo). Learning Resource Type descreve o tipo específico do OA, como por exemplo: exercício, simulação, questionário, etc. (ver Tabela 3) e, por fim, Interactivity Level que informa o nível de interatividade proporcionado pelo OA.

\subsection{Ontologias e Web Semântica}

A web semântica é a parte da web que provê técnicas, tecnologias e padrões que nos permitem atribuir significado às informações presentes na web. Dentre as suas tecnologias mais importantes está o Web Ontology Language [Shadbolt et al. 2006].

O conceito de ontologia em Inteligência Artificial (IA) é conhecido como o conjunto de entidades com suas relações, restrições, axiomas e vocabulário [Freitas 2003]. Dentre os benefícios de se utilizar ontologias, podemos destacar a utilização de uma linguagem declarativa [Sant'Anna et al. 2008]. Em geral, as ontologias são representadas em arquivos XML descritas em uma linguagem chamada Web Ontology Language (OWL).

Numa ontologia OWL pode-se incluir: "relações de taxonomia entre classes; propriedades dos tipos de dados e descrições dos atributos de elementos das classes, propriedades do objeto e descrições das relações entre elementos das classes, instâncias das classes e propriedades" [Santos and de Carvalho 2007]. 
V Congresso Brasileiro de Informática na Educação (CBIE 2016)

Anais do XXVII Simpósio Brasileiro de Informática na Educação (SBIE 2016)

Freitas 2003 destaca ainda outras vantagens de se utilizar Ontologia. Ontologias são, portanto, interessantes de serem utilizadas em sistemas que tem como foco o ensino, por auxiliarem na representação do conhecimento de modo formal e completo, além de ser um conceito maduro e possuir ferramentas bem trabalhadas que auxiliam a empregar o conceito.

Com o uso de Ontologias é possível ainda modelar regras em linguagens próprias, como a SWRL [Horrocks et al. 2004] e a SQWRL [O'Connor and Das 2009]. A linguagem SWRL foi elaborada para a criação de regras lógicas de inferência, sendo munida de operadores comuns da lógica proposicional com algumas limitações, como a falta dos operadores "OR"e "NOT".

Já o SQWRL foi criado com a intenção de trazer algumas funções do SQL convencional, tendo então como objetivo, criar queries de pesquisa dentro das ontologias. $\mathrm{O}$ SQWRL possui também a capacidade de receber algumas extensões, denominadas builtins, que acrescentam novas funcionalidades a linguagem.

\section{Abordagem Proposta}

Este trabalho propõe uma ontologia capaz de, a partir de metadados de OAs, gerar uma representação vetorial dos OAs considerando os EAs que atendem, para então, de forma eficiente, recomendar os OAs aos estudantes considerando seus EAs específicos. Inicialmente, foi analisado o mapeamento dos campos do padrão IEEE LOM para os EA de Felder, apresentado em [Dorça et al. 2016].

A Tabela 2 apresenta a base de regras que permite determinar quais EAs satisfazem determinado OA. As regras estão descritas em formato Prolog e observando-as, é possível notar que cada valor de um campo de determinado OA, representado pela variável $\mathrm{X}$, é relacionado a um EA. Como um exemplo, se o OA possui figure no campo Learning Resource Type, sabemos que este objeto pode ser melhor aproveitado por estudantes que possuem estilo Visual.

Tabela 2. Tabela de mapeamento entre os elementos IEEE LOM para os Estilos de Aprendizagem Felder-Silverman.

\begin{tabular}{|c|c|c|c|c|c|}
\hline Structure & Format & $\begin{array}{c}\text { Interactivity Type } \\
\text { (IT) }\end{array}$ & Learning & $\begin{array}{l}\text { ource Type } \\
\text { Г) }\end{array}$ & $\begin{array}{l}\text { Interactivity Level } \\
\text { (IL) }\end{array}$ \\
\hline $\begin{array}{l}\text { 1:Sequential(X):- } \\
\text { Structure(X,"atomic""); } \\
\text { Structure(X,"linear"). } \\
\text { 2:Global(X):- } \\
\text { Structure(X,"collection"); } \\
\text { Structure(X,"networked"); } \\
\text { Structure(X,"hierarchical"). }\end{array}$ & $\begin{array}{l}\text { 3:Visual(X):- } \\
\text { Format(X,"video"); } \\
\text { Format(X,"image"); } \\
\text { 4:Verbal(X):- } \\
\text { Format(X,"text"); } \\
\text { Format(X,"audio"); } \\
\text { 5:Active(X):- } \\
\text { Format(X,"application"). } \\
\text { 6:Reflective(X):- } \\
\text { Format(X,"video"); } \\
\text { Format(X,"audio"); } \\
\text { Format(X,"image"). }\end{array}$ & $\begin{array}{l}\text { 7:Active(X):- } \\
\text { IT(X,"active"); } \\
\text { IT(X,"mixed"). } \\
\text { 8:Reflective(X):- } \\
\text { IT(X,"expositive"); } \\
\text { IT(X,"mixed"). }\end{array}$ & $\begin{array}{l}\text { 9:Active(X):- } \\
\text { LRT(X,"exercise"); } \\
\text { LRT(X,"simulation"); } \\
\text { LRT(X,"questionnaire"); } \\
\text { LRT(X,"exam"); } \\
\text { LRT(X,"experiment"); } \\
\text { LRT(X,"problem statem."); } \\
\text { LRT(X,"self assessment"). } \\
\text { 10:Reflective(X):- } \\
\text { LRT(X,"diagram"); } \\
\text { LRT(X,"figure"); } \\
\text { LRT(X,"graph"); } \\
\text { LRT(X,"index"); } \\
\text { LRT(X,"slide"); } \\
\text { LRT(X,"table"); } \\
\text { LRT(X,"narrative text"); } \\
\text { LRT(X,"lecture"). } \\
\text { 11:Sensitive(X):- } \\
\text { LRT(X,"simulation"); } \\
\text { LRT(X,"figure"); } \\
\text { LRT(X,"graph"); } \\
\text { LRT(X,"index"); } \\
\text { LRT(X,"table"); } \\
\text { LRT(X,"experiment"); } \\
\text { LRT(X,"self assessment"). }\end{array}$ & $\begin{array}{l}\text { 12:Intuitive(X):- } \\
\text { LRT(X,"diagram"). } \\
\text { 13:Visual(X):- } \\
\text { LRT(X,"diagram"); } \\
\text { LRT(X,"figure"); } \\
\text { LRT(X,"graph"); } \\
\text { LRT(X,"experiment"). } \\
\text { 14:Verbal(X):- } \\
\text { LRT(X,"exercise"); } \\
\text { LRT(X,""uestionnaire"); } \\
\text { LRT(X,"index"); } \\
\text { LRT(X,"table"); } \\
\text { LRT(X,"narrative text"); } \\
\text { LRT(X,"exam"); } \\
\text { LRT(X,"problem statem."); } \\
\text { LRT(X,"self assessment"); } \\
\text { LRT(X,"lecture"). }\end{array}$ & $\begin{array}{l}\text { 15:Active(X):- } \\
\text { IL(X,"medium"); } \\
\text { IL(X,"high"); } \\
\text { IL(X,"very high"). } \\
\text { 16:Reflective(X):- } \\
\text { IL(X,"very low"); } \\
\text { IL(X,"low"); } \\
\text { IL(X,"medium"). }\end{array}$ \\
\hline
\end{tabular}


V Congresso Brasileiro de Informática na Educação (CBIE 2016)

Anais do XXVII Simpósio Brasileiro de Informática na Educação (SBIE 2016)

A partir da base de regras apresentada na Tabela 2, foi criada uma ontologia que possui duas classes. A primeira classe modela os OAs e a segunda os EAs, como é apresentado na Figura 1.

Figura 1. Representação gráfica da ontologia criada.

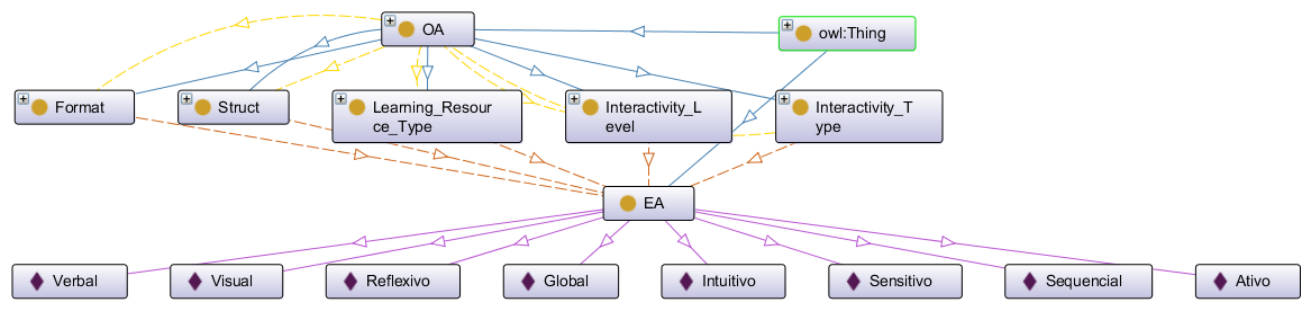

A classe OA possui cinco subclasses representando as 5 categorias do padrão utilizadas no mapeamento. Cada subclasse é composta por indivíduos que representam os valores que cada campo pode receber de acordo com o padrão IEEE LOM. Já a classe EA é composta apenas por indivíduos, onde cada indivíduo representa um dos oito EAs de Felder. Um melhor detalhamento sobre as classes e indivíduos pertencentes à ontologia pode ser visto na Tabela 3 .

Tabela 3. Classes e indivíduos pertencentes a Ontologia.

\begin{tabular}{|c|c|c|}
\hline Classe & Subclasses & Indivíduos disponíveis \\
\hline EA & Não Possui & Ativo, Reflexivo, Intuitivo, Sensitivo, Visual, Verbal, Sequencial, Global \\
\hline \multirow{5}{*}{ OA } & Structure & collection, hierarchical, linear, networked \\
\hline & Format & application, audio, image, text, video \\
\hline & Interactivity Type & active, expositive, mixed \\
\hline & Interactivity Level & very_high, high, medium, low, very_low \\
\hline & Learning Resource Type & $\begin{array}{c}\text { diagram, exam, exercise, experiment, figure, graph, index, lecture, } \\
\text { narrative_text, problem_statement, questionnaire, self_assessment, } \\
\text { simulation, slide, table }\end{array}$ \\
\hline
\end{tabular}

Além disso, foram criados dois Object Property(relacionamentos entre conceitos) para a Ontologia: o has e o is. Que correspondem a:

- has: Seu domínio são os conceitos da classe OA e possui como intersecções aqueles pertencentes às suas subclasses. Os OAs que a ontologia recebe, possuem os valores de seus campos representados com o uso deste Object Property, portanto, para dizer que um OA possui o valor "video"em seu campo Format basta dizermos a Ontologia: "has $(O A$, video $) "$. Na Figura 1 este relacionamento é representado por flechas tracejadas de cor amarela.

- is: Este relacionamento tem como domínio os conceitos pertencentes às subclasses da classe OA e possuem intersecções com os da classe EA. Este Object Property é utilizado na inferência dos EAs para cada valor pertencente aos OAs. Este relacionamento é destacado na Figura 1 através das flechas tracejadas de cor alaranjada.

A partir da ontologia, foi possível modelar regras de inferência na linguagem SWRL que identifiquem a quais EA determinado OA são aproveitados com maior eficiência. 
V Congresso Brasileiro de Informática na Educação (CBIE 2016)

Anais do XXVII Simpósio Brasileiro de Informática na Educação (SBIE 2016)

Na Tabela 4 podemos observar algumas regras como exemplo do total de cinquenta e nove construídas. Devido as limitações da linguagem, como a falta do operador de disjunção lógica, foram geradas muitas regras, porém simples. As regras foram modeladas de forma a identificar a quais EAs se relacionam os conceitos apresentados pelos OAs e não com o OA propriamente dito, através do Object Property "is".

Tabela 4. Exemplos de regras SWRL construídas

\begin{tabular}{|c|c|}
\hline Regra SWRL & Descrição \\
\hline has $(? \mathrm{o}$, collection $)->$ is (collection, Global $)$ & O atributo collection (campo Struct) é mapeado para EA Global \\
\hline has(?o, table) $->$ is (table, Sensitivo) & O atributo table (campo LRT) é mapeado para EA Sensitivo \\
\hline has(?o, table) ->is(table, Verbal) & O atributo table (campo LRT) é mapeado também para EA Verbal \\
\hline has(?o, table) ->is(table, Reflexivo) & O atributo table (campo LRT) é mapeado também para EA Reflexivo \\
\hline has(?o, video) ->is(video, Visual) & O atributo video (campo Format) é mapeado para EA Visual \\
\hline has(?o, video) ->is(video, Reflexivo) & O atributo video (campo Format) é mapeado também para EA Reflexivo \\
\hline has(?o, mixed) ->is(mixed, Ativo) & O atributo mixed (campo IT) é mapeado para EA Ativo \\
\hline has(?o, mixed) ->is(mixed, Reflexivo) & O atributo mixed (campo IT) é mapeado também para EA Reflexivo \\
\hline has $(?$ o, medium $)->$ is(medium, Ativo) & $\mathrm{O}$ atributo medium (campo IL) é mapeado para EA Ativo \\
\hline has $(?$ o, medium $)->$ is(medium, Reflexivo $)$ & O atributo medium (campo IL) é mapeado também para EA Reflexivo \\
\hline
\end{tabular}

LRT - Learning Resource Type

IT - Interactivity Type

IL - Interactivity Level

Foram modeladas regras SQWRL para realizar uma contagem de quantas regras SWRL (Tabela 4) um OA satisfaz para cada EA do modelo de Felder, gerando um vetor de 8 dimensões. A este processo denominamos vetorização, onde no vetor gerado, cada dimensão apresenta a quantidade de regras de inferência satisfeitas pelo OA para determinado EA.

O motivo do Object property "is" usar em sua relação os campos do OA ao invés do objeto é para que essa contagem seja possível de ser realizada. Foi utilizada a linguagem SQWRL, pois ela nos permite realizar consultas sobre as inferências das regras SWRL. A Tabela 5 apresenta as regras criadas para essa função, cada regra correspondente a um EA.

Tabela 5. Regras SQWRL modeladas

\begin{tabular}{|c|c|}
\hline Regras SQWRL & Descrição da contagem \\
\hline $\mathrm{OA}(? \mathrm{o}) \wedge \mathrm{ID}(? \mathrm{o}$, "1”) ^has(?o, ?x) ^is(?x, Ativo $)$ & Valores relacionados a "Ativo"existem no OA de ID "1" \\
\hline OA(?o) ^ID(?o,"1") ^has(?o, ?x) ^is(?x, Reflexivo) ->sqwrl:count(?x) & Valores relacionados a "Reflexivo"existem no OA de ID "1" \\
\hline $\mathrm{OA}(? \mathrm{o}) \wedge \mathrm{ID}(? \mathrm{o}, " 1 ")$ ^has(?o, ?x) ^is(?x, Visual) -> sqwrl:count(?x) & Valores relacionados a "Visual"existem no OA de ID "1" \\
\hline $\mathrm{OA}(? \mathrm{o}){ }^{\wedge} \mathrm{ID}(? \mathrm{o}, " 1 ")$ ^has(?o, ?x) ^is(?x, Verbal) ->sqwrl:count(?x) & Valores relacionados a "Verbal"existem no OA de ID "1" \\
\hline $\mathrm{OA}(? \mathrm{o}) \wedge \mathrm{ID}(? \mathrm{o}, " 1 ")$, has(?o, ?x) is(?x, Intuitivo) ->sqwrl:count(?x) & Valores relacionados a "Intuitivo"existem no OA de ID "1" \\
\hline OA(?o) ^ID(?o,"1") ^has(?o, ?x) is(?x, Sensitivo) ->sqwrl:count(?x) & Valores relacionados a "Sensitivo"existem no OA de ID "1", \\
\hline $\mathrm{OA}(? \mathrm{o}) \wedge \mathrm{ID}(? \mathrm{o}, " 1 ") \wedge$ has $(? \mathrm{o}$, ?x $)$ is(?x, Sequencial) ->sqwrl:count(?x) & Valores relacionados a "Sequencial"existem no OA de ID "1" \\
\hline $\mathrm{OA}(? \mathrm{o}){ }^{\wedge} \mathrm{ID}(? \mathrm{o}$, , 1 ") ^has(?o, ?x) ^is(?x, Global) -> sqwrl:count(?x) & Valores relacionados a "Global"existem no OA de ID "1" \\
\hline
\end{tabular}

\section{Experimento}

No experimento demonstrado foi utilizado um exemplo de OA apresentado pela Tabela 6. Nela, podemos observar um OA que possui os atributos dos OAs apresentados na primeira coluna, da mesma forma como são incluídos na ontologia, que equivalem a valores pertencentes aos campos do IEEE LOM apresentados na segunda coluna. 
V Congresso Brasileiro de Informática na Educação (CBIE 2016)

Anais do XXVII Simpósio Brasileiro de Informática na Educação (SBIE 2016)

Tabela 6. Exemplo de Objeto de Aprendizagem na Ontologia

\begin{tabular}{ll}
\hline Atributos & Campos IEEE LOM \\
\hline has(OA1, mixed) & Interactivity Type \\
has(OA1, collection) & Struct \\
has(OA1, video) & Format \\
has(OA1, table) & Learning Resource Type \\
has(OA1, medium) & Interactivity Level \\
\hline
\end{tabular}

Na Tabela 4, observamos quais inferências serão geradas. A Tabela 7 apresenta o resultado destas inferências, onde a primeira coluna referencia os atributos do OA incluídos na ontologia e a segunda os relacionamentos resultantes. A Tabela 8 apresenta a representação vetorial ou vetorização do OA apresentado na Tabela 6. Este é o resultado da contagem da quantidade de regras da Tabela 2 que foram satisfeitas pelo OA considerando cada EA atendido.

Tabela 7. Resultado das inferências da Ontologia

\begin{tabular}{|l|l|}
\hline Conceito de OA & Resultado da inferência \\
\hline has(OA1, mixed) & is(mixed, Ativo) \\
\hline has(OA1, collection) & is(collection, Global) \\
\hline has(OA1, video) & is(video, Visual) \\
\hline has(OA1, table) & is(video, Reflexivo) \\
\hline has(OA1, medium) & is(table, Verbal) \\
\hline is(table, Reflexivo) \\
\hline
\end{tabular}

Tabela 8. OA1 Vetorizado.

\begin{tabular}{cccccccc}
\hline \multicolumn{10}{c}{ OA1 } \\
\hline Ativo & Reflexivo & Visual & Verbal & Sequencial & Global & Intuitivo & Sensitivo \\
2 & 4 & 1 & 1 & 0 & 1 & 0 & 1 \\
\hline
\end{tabular}

O resultado obtido pelo processo é o esperado e, para cada EA dentro das quatro dimensões, pode-se também identificar a qual das 16 combinações possíveis de EA o objeto se encaixa. No exemplo mostrado na Tabela 8 observamos um OA melhor aproveitado por um aprendiz que seja pertencente ao grupo: Reflexivo/Visual/Global/Sensitivo, podendo então este OA ser facilmente recomendado a aprendizes que apresentam este perfil através de abordagens como a de [Carvalho et al. 2014].

\section{Considerações Finais}

Este trabalho apresentou uma ontologia que modela a relação entre OAs e EAs, de forma a oferecer suporte aos processos de recomendação personalizada de conteúdo. Esta On- 
V Congresso Brasileiro de Informática na Educação (CBIE 2016)

Anais do XXVII Simpósio Brasileiro de Informática na Educação (SBIE 2016)

tologia permite representar o conhecimento sobre os OA em relação aos EA que atendem de forma eficiente, e então realizar inferências sobre os OA, gerando representações vetoriais que podem ser facilmente utilizadas para descobrir o quanto um OA atende às preferências de determinado estudante.

A ontologia foi validada a partir de experimentos, e resultados promissores foram obtidos, demonstrando o potencial de contribuição desta proposta às abordagens para recomendação personalizada de conteúdo em sistemas educacionais.

Em um trabalho futuro será implementado um sistema de recomendação completo, que contemplará desde a entrada dos metadados e modelo de estudante até o ranqueamento dos OAs a serem apresentados aos estudantes. Este sistema será implantado e testado no Classroom eXperience [Ferreira et al. 2012], permitindo, assim, testes com OAs e estudantes reais.

\section{Agradecimentos}

Os autores agradecem as agências brasileiras de pesquisa CNPq, CAPES, FAPEMIG e a PROPP/UFU pelo apoio concedido a este trabalho.

\section{Referências}

Borenstein, N. S. and Freed, N. (1996). Multipurpose internet mail extensions (mime) part one: Format of internet message bodies.

Carvalho, V. C., Dorça, F. A., Cattelan, R. G., and Araújo, R. D. (2014). Uma Abordagem para Recomendação Automática e Dinâmica de Objetos de Aprendizagem Baseada em Estilos de Aprendizagem. In Anais do Simpósio Brasileiro de Informática na Educação, volume 25, page 1188 .

Dorça, F. A., Araújo, R. D., De Carvalho, V. C., Resende, D. T., and Cattelan, R. G. (2016). An Automatic and Dynamic Approach for Personalized Recommendation of Learning Objects Considering Students Learning Styles: An Experimental Analysis. Informatics in Education, 15(1):45-62.

Dorça, F. A., Lima, L. V., Fernandes, M. A., and Lopes, C. R. (2013). A New Approach to Discover Students Learning Styles in Adaptive Educational Systems. Revista Brasileira de Informática na Educação, 21(1):25-2013.

Dorça, F. A. and Resende, D. T. (2015). Recomendação de conteúdo personalizada com base em estilos de aprendizagem: uma abordagem prática. Revista Brasileira de Informática na Educação, 23(03):12.

Felder, R. M. and Silverman, L. K. (1988). Learning and teaching styles in engineering education. Engineering education, 78(7):674-681.

Ferreira, H. N. M., Araujo, R. D., de Amo, S. A., and Cattelan, R. G. (2012). Classroom Experience: A Platform for Multimedia Capture and Access in Instrumented Educational Environments. In 2012 Brazilian Symposium on Collaborative Systems, pages 59-64. IEEE.

Ferreira, L. G. A., Barbosa, J. L. V., Gluz, J. C., and Vicari, R. (2015). UbiGroup: Um Modelo de Recomendação Ubíqua de Conteúdo para Grupos Dinâmicos de Aprendizes. Revista Brasileira de Informática na Educação, 23(03):40. 
V Congresso Brasileiro de Informática na Educação (CBIE 2016)

Anais do XXVII Simpósio Brasileiro de Informática na Educação (SBIE 2016)

Franzoni, A. L., Assar, S., Defude, B., and Rojas, J. (2008). Student learning styles adaptation method based on teaching strategies and electronic media. In Advanced Learning Technologies, 2008. ICALT'08. Eighth IEEE International Conference on, pages 778-782. IEEE.

Freitas, F. L. G. (2003). Ontologias ea web semântica. Sociedade Brasileira de Computação (SBC). II Jornada de Mini-Cursos de Inteligência Artificial, pages 1-52.

Gluz, J. C. and Vicari, R. M. (2011). Uma Ontologia OWL para Metadados IEEELOM, DublinCore e OBAA. Anais do Simpósio Brasileiro de Informática na Educação, 1(1).

Graf, S., Liu, T.-C., Kinshuk, Chen, N.-S., and Yang, S. J. (2009). Learning styles and cognitive traits - Their relationship and its benefits in web-based educational systems. Computers in Human Behavior, 25(6):1280-1289.

Graf, S., Viola, S. R., Leo, T., and Kinshuk (2007). In-depth analysis of the feldersilverman learning style dimensions. Journal of Research on Technology in Education, 40(1):79-93.

Gruber, T. (1993). What is an ontology. WWW Site http://www-ksl. stanford. edu/kst/whatis-an-ontology. html (accessed on 07-09-2004).

Horrocks, I., Patel-Schneider, P. F., Boley, H., Tabet, S., Grosof, B., Dean, M., et al. (2004). Swrl: A semantic web rule language combining owl and ruleml. W3C Member submission, 21:79.

IEEE (2002). 1484.12.1-2002 IEEE Standard for Learning Object Metadata.

O'Connor, M. J. and Das, A. K. (2009). Sqwrl: A query language for owl. In OWLED, volume 529.

Rezende, P. A. A., Pereira, C. K., Campos, F., David, J. M. N., and Braga, R. (2015). PERSONNA: proposta de ontologia de contexto e perfil de alunos para recomendação de objetos de aprendizagem. Revista Brasileira de Informática na Educação, 23(01):70.

Sant'Anna, F., Cerqueira, R., and Soares, L. F. G. (2008). Nclua: objetos imperativos lua na linguagem declarativa ncl. In Proceedings of the 14th Brazilian Symposium on Multimedia and the Web, pages 83-90. ACM.

Santos, F. C. and de Carvalho, C. L. (2007). Aplicações de suporte à web semântica.

Shadbolt, N., Hall, W., and Berners-Lee, T. (2006). The semantic web revisited. Intelligent Systems, IEEE, 21(3):96-101.

Vicari, R. M., Bez, M., da Silva, J. M. C., Ribeiro, A., Gluz, J. C., Passerino, L., Santos, E., Primo, T., Rossi, L., Bordignon, A., et al. (2010). Proposta brasileira de metadados para objetos de aprendizagem baseados em agentes (obaa).

Weibel, S., Kunze, J., Lagoze, C., and Wolf, M. (1998). Dublin core metadata for resource discovery. Technical report.

Wiley, D. (2002). Connecting learning objects to instructional design theory: A definition, a metaphor, and a taxonomy, pages 3-23. The Agency for Instructional Technology, Bloomington, Indiana, USA. 